\title{
A SUBJECTIVE APPROACH TO QUANTUM PROBABILITY
}

\author{
EHUD LEHRER AND ERAN SHMAYA
}

June 21, 2021

\begin{abstract}
A likelihood order is defined over linear subspaces of a finite dimensional Hilbert space. It is shown that such an order that satisfies some plausible axioms can be represented by a quantum probability in two cases: pure state and uniform measure.
\end{abstract}

\section{INTRODUCTION}

According to the subjective approach probabilities are merely degreesof-belief of a rational agent. These degrees-of-belief might be indicated by the agent's willingness to bet or take other actions (see [6]). Savage (15]) derives both probabilities and utilities from rational preferences (i.e., that satisfy some putative properties) alone. Such preferences induce, in particular, a preference order over events. That is, an agent who holds rational preferences could indicate which of two events is more likely, and moreover, this likelihood order is transitive. Savage's first step is to derive a (finitely additive) probability that represents the likelihood order.

In this paper we adopt a similar approach and apply it to the quantum framework without going beyond probabilities. While classical probability is defined over subsets (events) of a state space, quantum probability is defined over subspaces of Hilbert space. Furthermore, disjointness of the classical model is replaced by orthogonality.

Formally, let $\mathcal{H}$ be a separable Hilbert space. A quantum probability measure $\mu$ over $\mathcal{H}$ assigns a number between 0 and 1 to every closed subspace that satisfies $\mu(A \oplus B)=\mu(A)+\mu(B)$ whenever $A \perp B$ and $\mu(\mathcal{H})=1$. Gleason's Theorem (Gleason (1957)) states that, if $\operatorname{dim}(\mathcal{H}) \geq 3$ every quantum measure $\mu$ is induced by a self-adjoint nonnegative operator $T$ with trace 1 in the following way: $\mu(A)=$ $\operatorname{tr}\left(\Pi_{A} T\right)$ for every subspace $A$, where $\Pi_{A}$ is the orthogonal projection over $A$.

School of Mathematical Sciences, Tel aviv Universtity, Tel Aviv 69978, Israel. e-mails: lehrer@post.tau.ac.il ; gawain@post.tau.ac.il . 
We assume the existence of a likelihood order $\preceq$ over subspaces of a given finite-dimensional Hilbert space. The statement ' $A$ is less likely than $B$ in one's eyes' could be understood operationally: one would prefer betting that $B$ occurs than that $A$ occurs (in the corresponding physical measurements).

We say that the likelihood order $\preceq$ can be represented by a quantum probability $\mu$ if $A \preceq B$ if and only if $\mu(A) \leq \mu(B)$. The goal of the line of research presented here is to find plausible properties (axioms, in the jargon of decision theory), preferably rationality-motivated, that ensure that $\preceq$ is representable by a quantum probability. Such a representation would mean that the agent acts as if he has quantitative degrees-of-belief that obey the rules of quantum probability.

Throughout, it is assumed that $\preceq$ possesses three properties. The first is that every subspace is more likely than the zero-dimensional one. The second is that a subspace $B$ is more likely than $A$ if and only if $B+C$ is more likely than $A+C$, whenever $C \perp A$ and $C \perp B$. That is, adding or deleting a subspace which is orthogonal to both $A$ and $B$ would preserve the likelihood ratio.

The classical counterpart of the third property is a consequence of the second. However, in the quantum model it has to be explicitly assumed. It states that if $B$ is more likely than $A$, then the orthogonal complement of $B$ is less likely than that of $A$.

Savage ([15]) also assumes these three axioms but, in order to obtain a representation by a measure, he needs an additional, less motivated, property that concerns with the richness of the state space. This one dictates that the state space could be split into mutually disjoint arbitrarily small (with respect to the likelihood order) subsets. The lack of a quantum counterpart (in the case of a finite-dimensional Hilbert space) of such an Archimedean property makes our study completely different from that of Savage.

Our main results refer to likelihood orders that can be represented by two types of quantum measures. The first is the most important from a physical point of view. The probabilities of this type are called pure states and are of the form $\mu(A)=\left\|\Pi_{A}(p)\right\|^{2}$ for some unit vector $p \in \mathcal{H}$. That is, the probability of a subspace $A$ is the length squared of the projection of the vector $p$. By Gleason's Theorem these measures are the extreme points of the convex set of all quantum probabilities. We characterize the likelihood orders that can be represented by a quantum measure. 
The second main result characterizes the likelihood orders that can be represented by the uniform distribution, defined by $\mu(A)=\frac{\operatorname{dim}(A)}{\operatorname{dim}(\mathcal{H})}$. This is the only quantum measure that obtains a discrete set of values.

Subjective analysis of quantum probability has been treated in the literature by several authors. Deutsch (7], [18) assumes that an agent assigns a value to any possible outcome of any possible measurement. Deutsch's analysis hinges heavily on what he calls the 'principle of substitutibility', which is similar to the Independence Axiom of von Neumann and Morgenstern ([17]). Barnum et al. ([1]) criticized Deutsch's argument and showed that his proof relies on a tacit symmetry assumption. Wallace ([18]) followed the line of Deutsch ([7]) and tried to make his assumptions more plausible. Gyntelberg and Hansen ([10]) applied a general event-lattice theory (with axioms that resemble those of von Neumann and Morgenstern) to a similar framework.

Pitowsky ([13]) assumed that for every possible measurement the agent has a certain probability over the corresponding outcomes. From some natural axioms he derives the probabilistic structure over quantum mechanics. Caves et al. ([2]) assume that the agent has degrees-ofbelief that determine the odds under which he is willing to take a bet. Under the assumption that the agent cannot be attacked by a Dutch book, and an assumption about 'maximal information', they showed that these degrees-of-belief must be given by a pure state.

The main difference between the aforementioned approaches and ours is that we do not assume that the agent has quantitative assignments: neither probabilities (i.e., numerical degrees-of-belief) to subspaces nor values to games or lotteries. Rather, the primitive of our model is a qualitative belief given by the likelihood order.

The paper is structured as follows. The next section characterizes the likelihood orders that admit a quantum probability representation in terms of continuity and a duality-like condition, called the cancelation

condition. Section 3 introduces the main axioms. Sections 4 and 5 are devoted to the main results: representation by a pure state and by a uniform distribution. Section [6] provides an example of a likelihood order that satisfies the main axioms except for continuity, and cannot be represented by a quantum measure. The paper is concluded with Section 7 that provides some final comments and open problems.

\section{The Cancelation Condition and Continuity}

Let $\mathcal{H}$ be a finite dimensional Hilbert space and let $\preceq$ be a weak order over linear subspaces of $\mathcal{H}$, that is $\preceq$ is reflexive (i.e., for every 
$A, A \preceq A$ ), transitive (i.e., for every $A, B, C$, if $A \preceq B$ and $B \preceq C$ then $A \preceq C$ ) and complete (i.e., for every $A, B, A \preceq B$ or $B \preceq A$ or both). We call $\preceq$ the likelihood order, and when $A \preceq B$ we say that $B$ is more likely than $A$. Denote by $\sim$ the equivalence relation induced by $\preceq$ (i.e., $A \sim B$ if $A \preceq B$ and $B \preceq A$ ) and by $\prec$ the corresponding strict order (i.e., $A \prec B$ if $A \preceq B$ and $B \npreceq A$.)

2.1. The cancelation condition. Cancelation condition (see, for example [8] is a well-known property of a weak order in the classical framework:

Proposition 1. Let $\preceq$ be a weak order over subsets of a finite set $\Omega$. For $A \subseteq \Omega$, denote by $\mathbf{1}_{A}$ the indicator function of $A$. Then there exists an additive probability measure $\mu$ over $\Omega$ such that $A \preceq B \leftrightarrow \mu(A) \leq$ $\mu(B)$ for every $A, B \subseteq \Omega$ if and only if the following conditions hold:

(1) For every $A \subseteq \Omega, \Phi \preceq A$.

(2) $\Phi \prec \Omega$.

(3) For every $n$, if $A_{1}, \ldots, A_{n}, B_{1}, \ldots, B_{n}$ are subsets of $\Omega$ such that $\sum_{i=1}^{n} \mathbf{1}_{A_{i}}=\sum_{i=1}^{n} \mathbf{1}_{B_{i}}$ and $A_{i} \preceq B_{i}$ for every $i$, then $A_{i} \sim B_{i}$ for every $i$.

Definition 1. Let $\mu$ be a quantum probability measure over $\mathcal{H}$. We say that $\mu$ represents $\preceq$ if $A \preceq B \leftrightarrow \mu(A) \leq \mu(B)$ for every two subspaces $A, B$ of $\mathcal{H}$.

In the quantum framework, orthogonal projection will replace the indicator functions that appear in Lemma 1.

Definition 2. The likelihood order $\preceq$ satisfies the cancelation condition if, for every $2 n$ subspaces of $\mathcal{H}, \overline{A_{1}}, \ldots, A_{n}, B_{1}, \ldots, B_{n}$, and $n$ positive numbers $\alpha_{i}, i=1, \ldots, n$, if $\sum_{i=1}^{n} \alpha_{i} \Pi_{A_{i}}=\sum_{i=1}^{n} \alpha_{i} \Pi_{B_{i}}$ and $A_{i} \preceq B_{i}$ for every $i=1, \ldots, n$, then $A_{i} \sim B_{i}$ for every $i=1, \ldots, n$.

2.2. Continuity. The cancelation condition by itself is not sufficient to ensure the existence of a representative measure (see Example 2 below.) Similar examples appear in the classical framework, when one tries to extend Proposition 1 to an infinite $\Omega$. In the current framework, in order to obtain a characterization of the likelihood orders that can be represented by a quantum measure, we need the additional assumption that $\preceq$ is continuous w.r.t. the natural topology over subspaces.

Let $A$ be subspace and $r$ be a positive number. Denote by $U$ the unit ball, $U=\{x ;\|x\| \leq 1\}$. By $N_{r}(A)$ we denote the $r$-neighborhood of $A$ restricted to the unit ball, $\cup\{V(x, r) ; x \in A$ and $x \in U\}$, where $V(x, r)$ is the ball of radius $r$ around $x$. For two subspaces $A$ and $B$ 
we denote $\delta_{*}(A, B)=\inf \left\{r>0: A \cap U \subset N_{r}(B)\right\}$ and $\delta^{*}(A, B)=$ $\inf \left\{r>0: B \cap U \subset N_{r}(A)\right\}$. The Hausdorff metric is defined as

$$
\delta(A, B)=\max \left\{\delta^{*}(A, B), \delta_{*}(A, B)\right\} .
$$

Definition 3. The likelihood order $\preceq$ is lower semi-continuous if, for every subspace $B$, the set of the subspaces $A$ such that $A \prec B$ is open with respect to $\delta$.

Theorem 1. Let $\preceq$ be a likelihood order. There exists a quantum probability measure that represents $\preceq$ if and only if the following conditions are satisfied:

(1) $\{0\} \preceq A$ For every subspace $A$ of $\mathcal{H}$;

(2) $\{0\} \prec \mathcal{H}$;

(3) $\preceq$ is lower semi-continuous;

(4) $\preceq$ satisfies the cancelation condition.

Proof. Assume first that $\preceq$ is represented by a quantum probability $\mu$. Then by Gleason's Theorem there exists a nonnegative operator $T$ with trace 1 such that $\mu(A)=\operatorname{tr}\left(\Pi_{A} T\right)$ for every subspace $A$ of $V$. In particular, the function $A \mapsto \mu(A)$ is continuous and therefore the order that it represents is lower semi-continuous. As for the cancelation condition, let $A_{1}, \ldots, A_{n}, B_{1}, \ldots, B_{n}$ be subspaces such that $\sum_{i=1}^{n} \alpha_{i} \Pi_{A_{i}}=\sum_{i=1}^{n} \alpha_{i} \Pi_{B_{i}}$ and $A_{i} \preceq B_{i}$, where $\alpha_{i}, i=1, \ldots, n$, are positive numbers It follows that

$$
\begin{aligned}
\sum_{i} \alpha_{i} \mu\left(A_{i}\right)= & \sum_{i} \alpha_{i} \operatorname{tr}\left(\Pi_{A_{i}} T\right)=\operatorname{tr}\left(\left(\sum_{i} \alpha_{i} \Pi_{A_{i}}\right) T\right)= \\
& \operatorname{tr}\left(\left(\sum_{i} \alpha_{i} \Pi_{B_{i}}\right) T\right)=\sum_{i} \alpha_{i} \operatorname{tr}\left(\Pi_{B_{i}} T\right)=\sum_{i} \alpha_{i} \mu\left(B_{i}\right) .
\end{aligned}
$$

Since $\mu\left(A_{i}\right) \leq \mu\left(B_{i}\right)$ for every $i$, it follows that $\mu\left(A_{i}\right)=\mu\left(B_{i}\right)$, which implies $A_{i} \sim B_{i}$.

Consider the finite dimensional Hilbert space of Hermitian operators over $\mathcal{H}$ with the inner product of two Hermitian operators $S$ and $T$ being $\operatorname{tr}(S T)$. Denote $\mathcal{C}=\operatorname{Conv}\left\{\Pi_{B}-\Pi_{A} ; A \prec B\right\}$ and $\mathcal{D}=\operatorname{span}\left\{\Pi_{B}-\Pi_{A} ; A \sim B\right\} . \mathcal{C}$ is a convex set and $\mathcal{D}$ is a linear subspace. From the cancelation condition it follows that $\mathcal{C}$ and $\mathcal{D}$ are disjoint.

The separation theorem (see, [14]) ensures that there is a non-zero linear function, represented in this case by the Hermitian operator $T$, such that $\operatorname{tr}(D T)=0$ for every $D \in \mathcal{D}$ (since $\mathcal{D}$ is a subspace) and $\operatorname{tr}(C T) \geq 0$ for every $C \in \mathcal{C}$. 
Since $\operatorname{tr}(D T)=0$ for $D \in \mathcal{D}$, it follows that $\operatorname{tr}(A T)=\operatorname{tr}(B T)$ if $A \sim B$. Let $A \prec B$. By the definition of $\mathcal{C}, \Pi_{A}-\Pi_{B} \in \mathcal{C}$. Due to lower semi-continuity, for $B^{\prime}$ sufficiently close to $B, A \prec B^{\prime}$ and therefore $\Pi_{A}-\Pi_{B^{\prime}} \in \mathcal{C}$. However, the set $\operatorname{Conv}\left\{\Pi_{A}-\Pi_{B^{\prime}}\right\}$, where $B^{\prime}$ is sufficiently close to $B$, contains $\Pi_{A}-\Pi_{B}$ as an interior point. Therefore, $\operatorname{tr}\left(\left(\Pi_{A}-\Pi_{B}\right) T\right)$ is strictly positive. We conclude that $T$ represents $\preceq$.

Finally, for every $A, \operatorname{tr}\left(\Pi_{A} T\right) \geq 0$ since $\{0\} \preceq A$. Therefore, $T$ is positive semidefinite. Moreover, since $\{0\} \prec \mathcal{H}$, it follows that $\operatorname{tr}(T)>$ 0 . Define $T^{\prime}=\frac{T}{\operatorname{tr}(T)}$. We obtain that $\preceq$ is represented by $\mu(A)=$ $\operatorname{tr}\left(T^{\prime} A\right), T^{\prime}$ is positive semidefinite and $\operatorname{tr}\left(T^{\prime}\right)=1$, as desired.

The cancelation condition (even in the classical framework) is difficult to justify. It is desirable to derive a probability representation of a likelihood order over linear subspaces from more plausible assumptions.

\section{De-FinetTi's And Other Axioms}

The most natural condition is de-Finetti's. When applied to classical probability it states that $C$ is disjoint of $A \cup B$, then $B$ is preferred to $A$ iff $B \cup C$ is preferred to $A \cup C$. In the quantum framework it takes following form:

de-Finetti's Axiom: For every linear subspaces $A, B, C$ of $\mathcal{H}$, if $A \perp C$ and $B \perp C$, then $A \preceq B$ iff $A+C \preceq B+C$.

In the classical framework it easily follows from de-Finetti's axiom that if $A \preceq B$ then $B^{c} \preceq A^{c}$. In the quantum framework, we need to require it explicitly.

Negation: For every two linear subspaces $A, B$ of $\mathcal{H}$, if $A \preceq B$ then $B^{\perp} \preceq A^{\perp}$.

As illustrated by the following example there might be weak orders that satisfy de-Finetti's axiom and not Negation.

Example 1. Let $\mathcal{H}$ be $\mathbb{R}^{2}$. Any monotonic (w.r.t set inclusion) weak order on $\mathcal{H}$ satisfies de-Finetti's axiom but it might not satisfy Negation. Let $\succeq^{\prime}$ be such a weak order. As for a higher dimensional Hilbert space, let $\mathcal{H}$ be $\mathbb{R}^{3}$. Let $p$ be the northern pole of the unit ball, $E$ be the equator, and define $\mu(u)=|\langle p, u\rangle|^{2}$ for any unit vector $u$.

Define $\preceq$ as follows: If $A$ and $B$ are two subspaces of different dimensions, then $A \succ B$ if the dimension of $A$ is greater than that of 
$B$. If $A=\operatorname{span}\{u\}$ and $B=\operatorname{span}\{v\}$, where $u$ and $v$ are unit vectors, then $A \succeq B$ either when $u \notin E$ and $\mu(u) \geq \mu(v)$ or when $u, v \in E$ and $u \succeq^{\prime} v$. Finally, if $A$ and $B$ are two-dimensional subspaces, then $A \succeq B$ either when $p \notin B$ and $\left\|\Pi_{A}(p)\right\|^{2}>\left\|\Pi_{B}(p)\right\|^{2}$ or when $p \in A \cap B$ and $A \cap E \succeq^{\prime} B \cap E$. The weak order $\preceq$ preserves de-Finetti's axiom but since $\succeq^{\prime}$ does not preserve Negation on $E$, so $\succeq$ does not on $\mathcal{H}$.

We will also need the obvious assumption that any subspace is as preferred as the zero-dimensional one. Formally,

Monotonicity: For every subspace $A$ of $\mathcal{H},\{0\} \preceq A$.

Note that Monotonicity and de-Finetti's axiom together imply that if $A \subseteq B$ then $A \preceq B$ for every pair $A, B$ of subspaces. Thus, $\preceq$ is monotonic with respect to set inclusion.

In the sequel, we will say that a weak order $\preceq$ satisfies the standard assumptions if it satisfies de-Finetti's axiom, Negation and Monotonicity.

Do the standard assumptions guarantee that $\preceq$ can be represented by a measure? The following is a counterexample.

Example 2. Let $\mu_{1}, \mu_{2}$ be two different quantum probability measures over $\mathcal{H}$ and define the lexicographic order induced by $\mu_{1}$ and $\mu_{2}$ as follows. $A \preceq B$ if either $\mu_{1}(A)<\mu_{1}(B)$ or $\mu_{1}(A)=\mu_{1}(B)$ and $\mu_{2}(A)<$ $\mu_{2}(B)$. Then, $\preceq$ satisfies the standard assumptions. Furthermore, it satisfies the cancelation condition.

The lexicographic order cannot be represented by a measure since it lacks the following property:

Separability: There is a countable set of subspaces, $\mathcal{A}$, such that for any two subspaces $B$ and $C$ such that $B \prec C$, there is $A \in \mathcal{A}$ that satisfies $B \preceq A \preceq C$.

As indicated by Debreu ([5]), Separability is necessary for $\preceq$ in order to be represented by a real function (not necessarily a measure).

\section{Pure States in $\mathbb{R}^{3}$}

The most important probabilities from the physical point of view are those of the form $\mu(A)=\left\|\Pi_{A}(p)\right\|^{2}$ for some unit vector $p \in A$. These distributions are sometimes called pure states. It follows from Gleason's Theorem that pure states are the extreme points of the convex set of all quantum probabilities. 
It is clear that if $\mu$ is a pure state and $\preceq$ is the induced likelihood order, then the one-dimensional subspace spanned by $p$ is equivalent (under $\sim$ ) to $\mathcal{H}$. In this section we prove the inverse statement. We say that $\preceq$ is non-trivial if there exists a subspace $A$ such that $\{0\} \prec A$.

Theorem 2. Let $\preceq$ be a weak order over subspaces of a finite dimensional real-Hilbert space that satisfies the standard assumptions and Separability. Assume that there exists a one-dimensional subspace $P$ such that $P \sim \mathcal{H}$. Let $p$ be a unit vector in $P$. If $\preceq$ is non-trivial, then $\preceq$ is represented by the pure state $\mu(A)=\left\|\Pi_{A}(p)\right\|^{2}$.

Proof. Let $E$ be the orthogonal complement of $P=\operatorname{span}\{p\}$. By Negation, since $P \sim \mathcal{H}$ it follows that $E \sim\{0\}$. Let $A$ be a subspace of $\mathcal{H}, A_{0}$ be $A_{0}=A \cap E$ and $A_{1}$ be the one-dimensional subspace of $\mathcal{H}$ that is spanned by $\Pi_{A}(p)$ (the orthogonal projection of $p$ over $A$.) Then, since $A_{0} \subset E$, it follows from Monotonicity that $A_{0} \sim 0$. Since $A_{1} \perp$ $A_{0}$ and $A=A_{1}+A_{0}$, it follows from de-Finetti's axiom that $A \sim A_{1}$. Thus, the likelihood order $\preceq$ is determined by its restriction to onedimensional subspaces. Moreover, since $\mu(A)=\mu\left(A_{1}\right)$, it is sufficient to prove that the likelihood order over one-dimensional subspaces is represented by $\mu$. Slightly abusing notation, we will identify a unit vector $u$ in $\mathcal{H}$ with the one-dimensional subspace spanned by $u$. With this convention, for every unit vector $u, \mu(u)=|\langle p, u\rangle|^{2}$.

Let $u, v$ be two unit vectors. We need to show that $u \preceq v$ iff $|\langle p, u\rangle|^{2} \leq|\langle p, v\rangle|^{2}$. By looking at the three-dimensional space $\mathcal{H}_{u v}$ spanned by $p, u, v$, with its two-dimensional subspace $\mathcal{H}_{u v} \cap E$ we can assume w.l.o.g. that $\operatorname{dim} \mathcal{H}=3$. In this case the theorem will follow directly from the following proposition. Let $S^{2}$ be the unit sphere in $\mathbb{R}^{3}$. We say that $\preceq$ is uniform if all the one-dimensional subspaces are equivalent.

Proposition 2. Let $\preceq$ be a weak order over $\mathbb{R}^{3}$ that satisfies the standard assumptions, and such that the restriction of $\preceq$ to one-dimensional subspaces is separable. Assume that $\preceq$ is not uniform and it attains its minimum over $S^{2}$ at $m$. Furthermore, assume that there exists a two-dimensional subspace $E$ such that $m \sim u$ for every $u \in E$. Let $p \in S^{2}$ such that $p \perp E$. Then, for every pair $u, v \in S^{2}, u \preceq v$ iff $|\langle p, u\rangle|^{2} \leq|\langle p, v\rangle|^{2}$.

Proof of Proposition 2. The proof of the proposition is broken into five claims. As usual, we identify elements of $S^{2}$ with their corresponding one-dimensional subspaces. 
Claim 1. Let $q, r \in S^{2}$ be such that $q \preceq r$. If $q^{\prime}$ and $r^{\prime}$ are, respectively, the orthogonal complements of $q$ and $r$ in the plane span $\{q, r\}$, then $r^{\prime} \leq q^{\prime}$

Proof. Let $n \in S^{2}$ such that $n \perp \operatorname{span}\{q, r\}$. Then $q^{\perp}=\operatorname{span}\left\{n, q^{\prime}\right\}$ and $r^{\perp}=\operatorname{span}\left\{n, r^{\prime}\right\}$. By Negation, $r^{\perp} \preceq q^{\perp}$. By de-Finetti's axiom, $r^{\prime} \preceq q^{\prime}$.

Claim 2. Let $u_{1}, u_{2} \in S^{2}$ be orthogonal vectors such that $u_{1} \sim u_{2} \sim m$. If $u \in \operatorname{span}\left\{u_{1}, u_{2}\right\}$, then $u \sim m$.

Proof. Note that $u_{2}$ is the orthogonal complement of $u_{1}$ in $\operatorname{span}\left\{u_{1}, u_{2}\right\}$. Let $u^{\prime}$ be the orthogonal complement of $u$ in $\operatorname{span}\left\{u_{1}, u_{2}\right\}$. Then $u_{1} \sim$ $m \preceq u^{\prime}$. By Claim 11. $u \preceq u_{2} \sim m$. Since $m \preceq u$, it follows that $u \sim m$.

Claim 3. Assume that there exists an orthogonal triple $u_{1}, u_{2}, u_{3}$ such that $m \sim u_{1} \sim u_{2} \sim u_{3}$. Then, $\preceq$ is uniform.

Proof. Let $v \in S^{2}$. Then there exists $u \in \operatorname{span}\left\{u_{1}, u_{2}\right\}$ such that $v \in \operatorname{span}\left\{u, u_{3}\right\}$. By Claim 2, $u \sim m$. But $u \perp u_{3}$ and therefore again by Claim 2, $v \sim m$.

For $q \in S^{2}$ we denote by $\theta(p, q)$ the angle between $p$ and $q$. Thus $0 \leq \theta(p, q) \leq \pi$ and $\cos \theta(p, q)=\langle p, q\rangle$. Let $N_{p}=\left\{q \in S^{2} \mid 0<\right.$ $\theta(p, q)<\pi / 2\}$ be the northern hemisphere relative to $p$, and $E_{p}=$ $\left\{q \in S^{2} \mid \theta(p, q)=\pi / 2\right\}=E \cap S^{2}$ be the equator relative to $p$. Let $q \in N_{p}$. Among the great circles which pass through $q$ there is a unique one that intersects $E_{p}$ in vector $x$ orthogonal to $q$. We follow Gleason (1957) and denote this circle by $\operatorname{EW}(q)$. Note that $q$ is the northern most point in $\operatorname{EW}(q)$ and that $\operatorname{EW}(q)$ is tangent to the latitude circle of $q$. We will need the following lemma, that appears in ([12]) (see also [4]).

Piron's Lemma. Let $q, r \in N_{p}$ such that $\theta(p, q)<\theta(p, r)$; then there exists a finite sequence $q=q_{0}, q_{1}, \ldots, q_{n}=r$ of points in $N_{p}$ such that $q_{i+1} \in \mathrm{EW}\left(q_{i}\right)$.

Claim 4. Under the assumption of Theorem 2, if $q, r \in N_{p}$ and $\mu(r)<$ $\mu(q)$, then $r \prec q$.

Proof. Note that $\mu(q)>\mu(r)$ iff $\theta(p, q)<\theta(p, r)$.

Let $q \in N_{p}$ and $q_{1} \in \operatorname{EW}(q)$. Let $q_{1}^{\prime} \in E W(q)$ be the orthogonal complement of $q_{1}$ in the plane of $\operatorname{EW}(q)$, and $q^{\prime} \in E_{p}$ be the orthogonal complement of $q$ in $\operatorname{EW}(q)$. Since $q^{\prime} \sim m \preceq q_{1}^{\prime}$, it follows from Claim 1 that $q_{1} \preceq q$. Moreover, $q_{1} \sim q$ only if $q_{1}^{\prime} \sim m$. By induction it follows from Piron's Lemma that $r \preceq q$. Furthermore, $r \sim q$ only if there exists 
$z \in N_{p}$ such that $z \sim m$. We prove that in this case $\preceq$ is uniform, which is excluded by assumption. This will complete the proof.

Note that for every $y$ such that $\theta(p, z)<\theta(p, y)<\pi-\theta(p, z), m \preceq$ $y \preceq z \sim m$. Thus, all the vectors in the band below $z$ are equivalent to $m$. We now show that one can find another point $p^{\prime}$, such that $x^{\prime} \sim m$ for every $x^{\prime} \in E_{p^{\prime}}$ and $\theta\left(p^{\prime}, z\right)=\frac{1}{2} \theta(p, z)$, and thus obtaining a wider band. By iterating this argument one can get wider and wider bands until one obtains a band that is wide enough to contain three orthogonal vectors. By Claim 3 , it would imply that $\preceq$ is uniform.

Let $p^{\prime}$ be point in $\operatorname{span}\{p, z\}$ for which $\theta\left(p, p^{\prime}\right)=\theta\left(p^{\prime}, z\right)=\frac{1}{2} \theta(p, z)$. It follows that, for every $x^{\prime} \in E_{p^{\prime}}, \frac{3}{2} \theta(p, z)<\theta\left(p, x^{\prime}\right)<\pi-\frac{3}{2} \theta(p, z)$. Thus, $E_{p^{\prime}}$ is entirely contained in the band defined by $p$ and $z$ and therefore $E_{p^{\prime}} \sim m$.

Claim 5. If $q, r \in N_{p}$ and $\mu(q)=\mu(r)$, then $q \sim r$.

Proof. We know from Claim 4 that for $q, r \in N_{p}$ such that $\mu(q)<\mu(r)$, $r \prec q$. Now suppose that there exist, for some $\alpha, 0<\alpha<\frac{\pi}{2}$, vectors $q_{0}, r_{0} \in N_{p}$ such that $q_{0} \prec r_{0}$ and $\mu\left(q_{0}\right)=\mu\left(r_{0}\right)=\alpha$. Let $Q=\{q \in$ $\left.S^{2} ; \mu(q)=\alpha, q \prec r_{0}\right\}$ and $R=\left\{r \in S^{2} ; \mu(r)=\alpha, q_{0} \prec r\right\}$. Then at least one of the sets $Q, R$ must be uncountable. Assume w.l.o.g. that $Q$ is uncountable. For every $q \in Q$, let $q^{\prime}, r^{\prime}$ be the orthogonal complements of $q, r_{0}$ resp. in $\operatorname{span}\left\{q, r_{0}\right\}$. It follows from Claim 1 that $r^{\prime} \prec q^{\prime}$. Notice moreover, that $\mu\left(q^{\prime}\right)=\mu\left(r^{\prime}\right)=1-\alpha-\mu(n(q))$ where $n(q) \in S^{2}$ is orthogonal to $\operatorname{span}\left\{q, r_{0}\right\}$. Since $\theta(p, n(q))=\cos \sqrt{\mu(n(q))}$ increases as $q$ approaches $r_{0}$ along the latitude circle of $r_{0}$, we get uncountable set of pairs $\left(r^{\prime}, q^{\prime}\right)$ such that $\mu\left(r^{\prime}\right)=\mu\left(q^{\prime}\right)$, but $r^{\prime} \prec q^{\prime}$ with different values of $\mu$ for different pairs. This, together with Claim 4 contradicts separability.

¿From Claims 4 and 5 it follows that $\mu(q) \leq \mu(r)$ iff $q \preceq r$, and therefore the proof of Proposition 2 is complete.

Back to the proof of Theorem 2. By assumption, $\preceq$ is non-trivial. Therefore, there exists a subspace $A$ which is strictly more likely than $\{0\}$. Suppose that $A$ is spanned by the orthogonal vectors $u_{1}, \ldots, u_{k}$.

Claim 6. At least one $u_{i}$ is strictly more likely than $\{0\}$.

Proof. Otherwise, $u_{i} \sim\{0\}$ for every $i=1, \ldots k$. By de-Finetti's axiom $\operatorname{span}\left\{u_{1}, u_{2}\right\} \sim \operatorname{span}\left\{u_{2}\right\} \sim\{0\}$. By successively adding the $u_{i}$ 's and by using de-Finetti's axiom one obtains that $A \sim\{0\}$, in contradiction with the assumption.

By Claim [6 we can assume that there is a vector $x \in S^{2}$ such that $x \succ\{0\}$. Let $y \in \operatorname{span}\{x, p\} \cap E$. Since $y \in E, y \sim\{0\}$. Furthermore, 
$y \perp p$. Let $x^{\prime}$ be the orthogonal complement of $x$ in $\operatorname{span}\{x, p\}$. Since $x^{\prime} \succ y$, by Claim $1 x \preceq p$. As $\preceq$ is an order, $\{0\} \prec x \preceq p$, and thus, $\{0\} \prec p$. This implies that $\preceq$, when restricted to $\mathcal{H}_{u v}$, is not uniform, as assumed by Proposition 2 . This enables us to use this proposition in order to complete the proof of Theorem 2

Remark 1. No sort of continuity is assumed in Theorem 2 . Nevertheless, $\preceq$ is represented by a measure and is therefore continuous.

\section{THE UNIFORM MEASURE}

The only quantum probability measures over a finite dimensional Hilbert space $\mathcal{H}$ which receives discrete values is given by the uniform measure, $\mu(A)=\frac{\operatorname{dim}(A)}{\operatorname{dim}(\mathcal{H})}$. It turns out that this is the case characterized by the property that all one-dimensional subspaces are equally likely. Formally,

Proposition 3. Let $\preceq$ be a weak order over subspaces of a finite dimensional Hilbert space that satisfies de-Finetti's axiom. If all one-dimensional subspaces are equivalent, then either $\preceq$ is trivial (i.e. $\{0\} \sim A$ for every subspace $A$ of $\mathcal{H})$ or $\preceq$ is represented by the uniform measure.

Proof. Assume that every one-dimensional subspace is equivalent to some one-dimensional subspace, say, $m$. If $A_{1}, A_{2}$ are two-dimensional such that $A_{1} \cap A_{2}$ is one-dimensional, we can assume that $A_{1}=\operatorname{span}\left\{a_{0}, a_{1}\right\}$ and $A_{2}=\operatorname{span}\left\{a_{0}, a_{2}\right\}$ where $a_{0} \perp a_{1}$ and $a_{0} \perp a_{2}$. Since $a_{1} \sim a_{2} \sim m$ we get, by de-Finetti's axiom, that $A_{1} \sim A_{2}$. If $A_{1} \cap A_{2}=\{0\}$, we can find a two-dimensional subspace $A^{\prime}$ such that $A_{1} \cap A^{\prime}$ and $A_{2} \cap A^{\prime}$ are one-dimensional. Therefore $A_{1} \sim A^{\prime} \sim A_{2}$. Thus every two-dimensional subspaces are equivalent. By a similar argument, two subspaces of the same dimension are equivalent.

Finally, if $\{0\} \sim m$, it follows by de-Finetti's axiom that $\{0\} \sim \mathcal{H}$. If $0 \prec m$, then again by de-Finetti's axiom, if $A \perp m$ and $A^{\prime}=A+m$ then $A \prec A^{\prime}$. Using the equivalence of two subspaces with the same dimension, it follows that if $\operatorname{dim}\left(A^{\prime}\right)=\operatorname{dim}(A)+1$, then $A \prec A^{\prime}$ and therefore $\preceq$ is represented by $\mu(A)=\frac{\operatorname{dim}(A)}{\operatorname{dim}(\mathcal{H})}$.

The following nontrivial fact about quantum probabilities follows from Gleason's Theorem:

Proposition 4. Let $\mathcal{H}$ be a finite-dimensional Hilbert space and $\mu$ be a quantum probability over $\mathcal{H}$. Assume that there exist one-dimensional subspaces (not necessarily orthogonal) $u_{1}, \ldots, u_{n}$ of $\mathcal{H}$ such that $\mathcal{H}=$ 
$u_{1}+\cdots+u_{n}$ and $\mu\left(u_{1}\right)=\cdots=\mu\left(u_{n}\right) \leq \mu(x)$ for every one-dimensional subspace $x$ of $\mathcal{H}$. Then, $\mu$ is the uniform measure.

We show that this proposition is a consequence of the standard assumptions, with the additional assumption that $\preceq$ is continuous over one-dimensional subspaces.

Definition 4. The likelihood order $\preceq$ is continuous over one-dimensional subspaces if for every unit vector $v$ the sets $\{u ; u \prec v\}$ and $\{u ; v \prec u\}$ are open.

We note that if $\preceq$ is continuous over one-dimensional subspaces then its restriction to one-dimensional subspaces is also separable. Indeed, let $D \subseteq S^{2}$ be a countable dense set w.r.t. the Euclidean topology of $S^{2}$. For every $u, v \in S^{2}$ such that $u \prec v$, let $U=\left\{u^{\prime} \in S^{2} \mid u \prec u^{\prime}\right\}$ and $V=\left\{v^{\prime} \in S^{2} \mid v^{\prime} \prec v\right\}$. Since $S^{2}=U \cup V$ and $S^{2}$ is connected, $U \cap V \neq \phi$. As $D$ is dense, there exists $d \in D$ such that $d \in U \cap V$. Thus, $u \prec d \prec v$.

We state the result in $\mathbb{R}^{3}$. It can easily be extended to every finitedimensional Hilbert space.

Theorem 3. Let $\preceq$ be a weak order over $\mathbb{R}^{3}$ that satisfies the standard assumptions. Assume that $\preceq$ is continuous over one-dimensional subspaces. If $u_{1}, u_{2}, u_{3}$ is a basis (not necessarily orthogonal) that satisfies $u_{1} \sim u_{2} \sim u_{3} \sim m$, where $m$ is a minimum of $\preceq$, then $x \sim m$ for every $x \in S^{2}$.

The theorem is proved in a few steps. Denote by $M$ a maximum of $\preceq$.

Claim 7. Let $u, v \in S^{2}$ such that $u \sim m$ and $v \sim M$. Let $u^{\prime}, v^{\prime}$ be the orthogonal complements of $u, v$ in $\operatorname{span}\{u, v\}$, respectively. Then, $u^{\prime} \sim M$ and $v^{\prime} \sim m$.

Proof. Since $m \sim u \preceq v^{\prime}$ it follows from Claim 11 that $v \preceq u^{\prime}$. But $v \sim M$ and $M$ is a maximum. Therefore $u^{\prime} \sim M$. By a similar argument $v^{\prime} \sim m$.

Claim 8. If $u_{1} \perp u_{2} \in S^{2}$ and $m \sim u_{1} \sim u_{2}$, then either all onedimensional subspaces are equivalent or $\preceq$ is represented by a pure state.

Proof. Let $E=\operatorname{span}\left\{u_{1}, u_{2}\right\}$. By Claim 2, $u \sim m$ for every $u \in E$. By Proposition 2, either $\preceq$ is trivial or $\preceq$ is represented by a pure state.

Claim 9. If $u_{1} \neq \pm u_{2} \in S^{2}, M \sim u_{1} \sim u_{2}$ and $p \perp u_{1}, u_{2}$, then $p \sim m$. 
Proof. Let $x \in N_{p}$ be such that $x \sim m$ and $\theta(p, x)$ is minimal. If $x \neq p$ then $x$ cannot be orthogonal to both $u_{1}$ and $u_{2}$. Assume therefore w.l.o.g. that $\left\langle x, u_{1}\right\rangle \neq 0$. Let $u_{1}^{\prime}$ be the orthogonal complement of $u_{1}$ in the plane $\operatorname{span}\left\{x, u_{1}\right\}$. By Claim [7, $u_{1}^{\prime} \sim m$. Moreover $\theta\left(p, u_{1}^{\prime}\right)<$ $\theta(p, x)$ since $x \in E W\left(u_{1}^{\prime}\right)$. This contradicts the choice of $x$. It therefore follows that $x=p$, meaning that $p \sim m$.

Claim 10. If $m$ and $M$ are any minimal and maximal elements in $S^{2}$ and $m \prec M$ (i.e., $\preceq$ is not trivial), then $m \perp M$.

Proof. Assume the contrary. Let $a$ be the orthogonal complement of $m$ in $\operatorname{span}(m, M)$. By Claim $7 a \sim M$. Let $p$ satisfy $p \perp M$ and $p \perp a$. By Claim 9 $p \sim m$. However, since $m \in \operatorname{span}(M, a), p \perp m$. Therefore it follows from Claim 8 that $\preceq$ is represented by a pure state, in which case the claim holds.

We now turn to the proof of the Theorem 3 . Let $M$ be a maximal element. If $\preceq$ is not trivial then from the last claim it follows that $M \perp u_{i}$ for every $i$. This is impossible since since $u_{1}, u_{2}, u_{3}$ are linear independent, and the proof is complete.

Remark 2. We do not know whether Theorem 3 holds true without the assumption that $\preceq$ is continuous over one-dimensional spaces. The proof hinges on this assumption in two ways. First, in that $\preceq$ attains a minimum and a maximum. Second, in Claim $9 x$ is chosen so that among all $x \sim m, \theta(p, x)$ is minimal. While we could explicitly assume that $\preceq$ attains a minimum and a maximum, we could not dispose of the continuity assumption in the proof of Claim 9

\section{A Counterexample}

In this section we present an example of a separable (though not continuous) weak order over subspaces of $\mathbb{R}^{3}$ that satisfies the standard assumptions but does not admit a representation via a quantum measure. We need the following two lemmas.

Lemma 1. Let $\preceq$ be a weak order over one-dimensional subspaces of $\mathbb{R}^{3}$ such that for every two-dimensional subspace $U$ of $\mathbb{R}^{3}$ and every one-dimensional subspaces $u, v$ of $U$ one has $u \preceq v \longleftrightarrow v^{\prime} \preceq u^{\prime}$ where $u^{\prime}, v^{\prime}$ are the orthogonal complements of $u, v$ resp. in $U$, then $\preceq$ can be extended to a weak order over $\mathbb{R}^{3}$ that satisfies the standard assumptions.

Proof. We define $\preceq$ as follows. Let $U, V$ be two subspaces of $\mathbb{R}^{3}$. If $\operatorname{dim}(U)<\operatorname{dim}(V)$ then $U \prec V$. If $\operatorname{dim}(U)=\operatorname{dim}(V)=2$ then $U \preceq V$ iff $V^{\perp} \preceq U^{\perp}$. Negation is obviously satisfied. As for de-Finetti's 
axiom, let $u, v$ be two different one-dimensional subspaces and $x$ be the one-dimensional subspaces such that $x \perp u, v$. Let $u^{\prime}, v^{\prime}$ be the orthogonal complements of $u, v$ in $u+v$. Then $(x+u)^{\perp}=u^{\prime}$ and $(x+v)^{\perp}=v^{\prime}$. Since, by the assumption of the lemma $v^{\prime} \preceq u^{\prime}$, it follows by definition of $\preceq$ that $x+u \preceq x+v$.

The second lemma states that if $\preceq$ is represented by a probability measure, then the order over one-dimensional subspaces of a fixed twodimensional subspace $U$ has a very specific form. As usual we identify one-dimensional subspaces with unit vectors. If $S^{1}$ is the unit circle of $U$, the lemma essentially says that either all elements of $S^{1}$ are equivalent, or there is a single maximal element $x \in S^{1}$ that satisfies $y_{1} \preceq y_{2}$ iff $y_{2}$ is closer than $y_{1}$ to $x$.

Lemma 2. Let $\mu$ be a probability measure over $\mathbb{R}^{3}$ and $\preceq$ the corresponding weak order over subspaces. Let $U$ be a two-dimensional subspace of $\mathbb{R}^{3}$. Then, either all one-dimensional subspaces of $U$ are equivalent, or there exists unit vector $x \in U$ such that for every pair $y_{1}, y_{2}$ of unit vectors $y_{1} \preceq y_{2}$ iff $\left|\left\langle x, y_{1}\right\rangle\right| \leq\left|\left\langle x, y_{2}\right\rangle\right|$.

Proof. By Gleason's Theorem, there exists a positive semidefinite operator $T$ such that $\mu(A)=\operatorname{tr}\left(\Pi_{A} T\right)$. Consider the operator $\Pi_{U} T \Pi_{U}$. This is a positive semidefinite operator. Its spectral decomposition is of the form

$$
\Pi_{U} T \Pi_{U}=\alpha \Pi_{x}+\beta \Pi_{x^{\prime}}
$$

where $x, x^{\prime}$ are orthogonal eigenvectors in $U$ with corresponding eigenvalues $\alpha, \beta$ such that $\alpha+\beta=1$. We assume that $\alpha \geq \beta$. It follows that for every unit vector $y$ in $U$,

$$
\begin{aligned}
\mu(y)=\operatorname{tr}\left(\Pi_{y} T\right)= & \operatorname{tr}\left(\pi_{y} \Pi_{U} T \Pi_{U}\right)= \\
& =\alpha|\langle y, x\rangle|^{2}+\beta\left|\left\langle y, x^{\prime}\right\rangle\right|^{2}=\beta+(\alpha-\beta)\left|\langle y, x\rangle^{2}\right| .
\end{aligned}
$$

Thus, if $\alpha=\beta$ then all $y \in S^{2} \cap U$ are equivalent. If $\alpha>\beta$ then $\mu(y)$ is a monotonic function of $|\langle y, x\rangle|$.

Example 3. Let $\succeq^{\prime}$ be a weak order on one-dimensional subspaces of $\mathbb{R}^{2}$ that satisfies the condition of Lemma 1 but not the condition of Lemma 2. Define $\preceq$ on one-dimensional subspaces of $\mathbb{R}^{3}$ as follows: Let $p$ be the northern pole of the unit sphere in $\mathbb{R}^{3}$. Let $u$ and $v$ be unit vectors, then $u \succeq v$ either when $u \notin E$ and $\mu(u) \geq \mu(v)$ or when $u, v \in E$ and $u \succeq^{\prime} v$. By Lemma $1 \prec$ can be extended to a weak order over $\mathbb{R}^{3}$ that satisfies the standard assumptions. However, since the condition of Lemma 2 is not satisfied, $\preceq$ cannot be represented via a quantum measure. 


\section{Final Comments and Open Problems}

7.1. Representation and continuity. In Gleason's Theorem ([9]) continuity is not assumed and is a consequence of the existence of a frame function. When the primitive of the model is a likelihood order, matters are different. The likelihood order in Example 3 satisfies deFinetti's axiom, Negation, Monotonicity and Separability and cannot be represented by a quantum measure. This order, which is not continuous, suggests that continuity must be explicitly assumed and cannot be derived from more plausible assumptions.

The question whether every continuous likelihood order which satisfies de-Finetti's axiom, Negation, Monotonicity and Separability can be represented by a quantum measure is still open.

\subsection{Partial representation.}

Definition 5. We say that $\mu$ partially represents $\preceq$ if $A \preceq B \longrightarrow$ $\mu(A) \leq \mu(B)$ for every two subspaces $A, B$ of $\mathcal{H}$.

It turns out (we state without a proof) that if $\preceq$ satisfies the cancelation condition, then there exists a quantum probability measure that partially represents $\preceq$. Also, from the proof of Theorem 2 it follows that, if there exists a one-dimensional subspace $p$, such that $p \sim \mathcal{H}$, then (without assuming separability) $\preceq$ admits a partial representation by a pure state.

7.3. Qualitative additivity and discrete orders. Gleason's Theorem implies that the only quantum probability measure which obtains a discrete set of values is the uniform measure. The question arises whether the same is true for likelihood orders. We say that $\preceq$ is discrete if the restriction of $\sim$ to one-dimensional subspaces has only finitely many equivalence classes. For instance, if $\preceq$ is represented by the uniform probability, then its restriction to one-dimensional subspaces has only one equivalence class.

Kochen-Specker's Theorem ([11]) actually refers to likelihood orders whose restriction to one-dimensional subspaces have precisely two equivalence classes. In order to prove this result using likelihood orders terms only, one needs to strengthen de-Finetti's axiom are Negation. The following axiom is a consequence of de-Finetti's axiom in the classical case, but not in the a quantum set-up.

Qualitative additivity: Let $A_{1}, A_{2}, B_{1}, B_{2}$ be linear subspaces of $\mathcal{H}$ such that $A_{1} \perp A_{2}$ and $B_{1} \perp B_{2}$. If $A_{i} \preceq B_{i}, i=1,2$ then $A_{1} \oplus A_{2} \preceq$ $B_{1} \oplus B_{2}$. Furthermore, one strict likelihood on the former inequalities implies strict likelihood in the later inequality. 
Suppose that $A_{1}$ and $A_{2}$ are orthogonal and the same for the $B_{i}$ 's. Qualitative additivity states that, if the $A_{i}$ 's are at least as likely as $B_{i}$ 's, then the subspace spanned by the $A_{i}$ 's is at least as likely as that spanned by $B_{i}$ 's. That is, adding or subtracting a more likely subspace to or from a subspace which is already more likely, cannot make the outcome less likely.

Suppose that $\preceq$ is defined over $\mathbb{R}^{3}$ and there are only two equivalence classes of one-dimensional subspaces, say, green and red. If $\preceq$ satisfies Qualitative additivity, then in any orthogonal triple there is the same number of green representatives, and moreover, a two dimensional subspace spanned by uni-colored vectors contains only vectors of the same color. These are precisely the terms of Kochen-Specker's Theorem ([1] $)$. It states that there exists no likelihood order that satisfies Qualitative additivity and has precisely two one-dimensional equivalence classes.

This result suggests that the only discrete likelihood order that satisfies Qualitative additivity is that induced by the uniform measure.

\section{REFERENCES}

[1] Barnum H., C.M. Caves, J. Finkelstein, C.A. Fuchs and R. Schack (2000) Quantum probability from decision theory? Proc. R. Soc. Lond. A 456, 11751182.

[2] Caves C.M., C.A. Fuch and R. Schack (2002) Quantum probabilities as Bayesian probabilities. Physical Review A. 65, 022305.

[3] Chevalier G., A. Dvurecenkij and K. Svozil (2000) Piron's and Bell's Geometric Lemmas and Gleason's Theorem. Foundations of Physics 30, 1737-1755.

[4] Cooke, R., M. Keane, and W. Moran (1985) An Elementary proof of Gleason's Theorem.Proc.Cambr.Phil.Soc 98, 117-128.

[5] Debreu, G. (1954) Representation of a preference ordering by a numerical function. In Decision Processes, Thrall, R.M., Coombs, C.H. and Davis, R.L., Editors, Wiley, New York, 159-165.

[6] de-Finetti, B. (1974-5) Theory of Probabilty, (translation of 1970 book) 2 volumes, New York: Wiley.

[7] Deutsch, D. (1999) Quantum theory of probability and decisions. Proc. R. Soc. Lond. A 455, 3129-3137.

[8] Fishburn, P. (1999) Preference structures and their numerical representations. Theoret. Comput. Sci. 217, 359-383.

[9] Gleason, A.M. (1957) Measures on the closed subspaces of a Hilbert Space. Journal of Mathematics and Mechanics 6, 885-893.

[10] Gyntelberg, J. and F. Hansen (2004) Expected utility theory with "small worlds", http://www.econ.ku.dk/wpa/pink/2004/0420.pdf

[11] Kochen, S. and E. P. Specker (1967) The problem of hidden variables in quantum mechanics. Journal of Mathematics and Mechanics , 17, 59-87.

[12] Piron C. (1976), Foundations of Quantum Physics, Addison-Wesley, Mass. 
[13] Pitowsky, I. (2003) Betting on the outcomes of measurements. Studies In History and Philosophy of Modern Physics 34, 395-414.

[14] Rockefeller, R.T., (1970) Convex Analysis, Princeton University Press, Princeton.

[15] Savage, L.J. (1954) The Foundations of Statistics London: John Wiley and Sons.

[16] von Neumann, J. (1955) Mathematical Foundations of Quantum Mechanics. Princeton: Princeton University Press. (German Edition 1932).

[17] von Neumann, J. and O. Morgenstern (1944) Theory of games and economic behavior, Princeton University Press.

[18] Wallace, D. (2000) Quantum probability from decision theory, revisited. quantphi/02-11104. 